\title{
Numerical and Experimental Investigation of the Argillaceous Sandstone Mechanical Properties by the Discrete Element Method
}

\author{
Chengxu Xiao ${ }^{1,2, a}$,Shouchun Deng ${ }^{2, b}$, Zhenghong Huang ${ }^{2, c}$, Hong Zuo $2, \mathrm{~d}$ \\ ${ }^{1}$ Hubei University of Technology, Hubei 430068,China \\ 2Institute of Rock and Soil Mechanics, Chinese Academy of Sciences, Wuhan, Hubei 430071, \\ China \\ am15007165603@163.com, bdengshouchun@gmail.com, c517297348@qq.com, \\ d1260181575@qq.com
}

\begin{abstract}
Key words: Rock mechanics, Particle flow code, Mesoscopic parameters, Friction coefficient
Abstract. In this paper, the triaxial compression test of the argillaceous sandstone is carried out by using the digital control electrohydraulic servo testing machine (RMT-150C). Based on the results of the macroscopic test, we use the three-dimensional particle flow code to simulate the macroscopic stress and deformation characteristics of the argillaceous sandstone under the three-dimensional stress condition, and the meso-macroscopic parameters are connected to simulate the different porosity, The coefficient of friction, the bond strength on the specimen, and further improve the study of argillaceous sandstone. The results show that the simulation results of particle flow program are in good agreement with the macroscopic experiments, but the strength is slightly lower than the experimental value. The strength of argillaceous sandstone increases with the friction coefficient between the particles and the bond strength at the contact point. The strength decreases with the increase of the porosity ratio, but the effect is not as obvious as the friction coefficient. The significance of the study is to reveal the factors that reveeal the strength of coarse-grained soil from a microscopic point of view, breaking the limitations of conventional triaxial test.
\end{abstract}

\section{Introduction}

Argillaceous sandstone as the construction of underground engineering in one of the most common sedimentary rock, its mechanical properties has been of study and research of many experts and scholars.Hong-mei tang et al.[1] studied the change of elastic modulus, Poisson's ratio and compressive strength under different confining pressure conditions, and obtained the expression form of the second parabola of sandstone. Tie-lin Han et al. [2] conduct an triaxial experiment of three kinds of different stress paths, such as deformation characteristics, strength characteristics and failure characteristics, and its mechanism analysis, were carried out on the sandstone samples. The comparative analysis of the mechanical properties of sandstone samples is carried out. Xiliang Liu et al. [3] analyzed the effects of deep temperature and pressure conditions on the mechanical properties of fine sandstone, and revealed that there was a certain degree of nonuniformity in the strain after damage and failure. Song Han , Feng Caimei [4] systematically studied the influence of deep geostress conditions on the mechanical properties of different sandstones, and established the correlation between the mechanical properties of sandstone and deep in-situ stress. Gang Wu et al. [5] analyzed the changes of apparent morphology, peak stress, peak strain, elastic modulus, Poisson's ratio and stress-strain curve after sandstone heating. Anbin Zhang et al [6] studied the influence of water on the mechanical properties, failure modes and microstructure of rock fracture. Lu Wang et al. [7] analyzed the permeability and acoustic emission characteristics of sandstone during deformation and failure. The mechanical properties of sandstone are not only affected by the stress, temperature and water of the surrounding rock, but also related to the composition of the sandstone itself. The mechanical properties of the sandstone with different particle size are quite different. Kang Han [8] show that the strength and deformation characteristics of the two kinds of sandstone are analyzed by conventional triaxial compression test in sandstone with fine and medium sizes. Zou Hang [9] To investigate the influence of particle size on the mechanical behavior of sandstone and permeability 
characteristics. Xian-shan Liu , Ming Xu [10] Based on the numerical model of three-dimensional particle flow code, four numerical models of different particle size distributions were established to simulate the mechanical response of sandstone in shear process.

However, for a project with a certain design period or a long construction time and operation time, it is necessary to study the similarities and differences of the mechanical behavior of the sandstone with different particle size in the long and short term load. The above study has a certain significance for studying the effect of particle size on the mechanical properties of sandstone. We use the particle flow code to simulate the deformation properties of the granular polymer, construct the corresponding particle flow sandstone specimen model, and simulate the triaxial test of the sandstone. The results show that the particle flow code can simulate the indoor triaxial test of sandstone, especially the macroscopic and microscopic performance of the deformation of the shaly sandstone can give a good regularity. Numerical simulation of particle flow code through laboratory tests, adjustment of various parameters of the particles, and the test results obtained similar stress-strain curve, there is a certain theoretical analysis and practical value.

\section{Theory of Discrete Element Method}

The mechanical and hydrological behavior of shale is significantly affected by the nucleation and growth of micro cracks and interactions among micro fractures[11]. Microscopic information, such as the distribution of grain sizes, compositions and orientations, the spatial correlations between these grain characteristics, and the distribution and nature of pre-existing and load-induced fractures, have important impacts on macroscopic mechanical behaviors. Realistic and robust shale mechanical models should account for these details at both small and large scales.

In principle, shale can be approximated as assemblies of independent discrete elements (particles) of various sizes and material properties that interact via cohesive interactions (representing cementation along grain boundaries), repulsive forces, and frictional forces[11-13]. The macroscopic behavior can then be modeled as the collective behavior of many interacting discrete elements. This discrete (or distinct) element method (DEM) is particularly suitable for modeling shale-proppant mechanical interactions, where shale can be represented as grains (discrete elements with various sizes and properties) that are cemented into a cohesive body, and proppants can be modeled as the individual discrete particles with various sizes (following certain proppant size distribution-density function) that are not cemented together (no cohesive strength).

In the standard DEM models, the contact points between pairs of DEM particles are treated as mechanical bonds (such as springs, beams, or more complex elements) that carry both forces and moments. The contact forces (normal force and shear force) and moments (in-plane twist moment and out-of-plane bending moment) between DEM particles can be calculated in an incremental fashion. Then the movement of each DEM particle, including translation and rotation, can be calculated from Newton's second law through an explicit numerical integration in the fashion of molecular dynamics (MD). Dynamic fracturing process can be naturally modeled through a series of events breaking bonds between DEM particles.

Both the shale grains and "cement" bonds between shale grains are deformable, and the cement bonds may break and be removed from the system when either the tensile or shear stress exceeds its corresponding critical strength threshold values. As shown in Fig. 1, the DEM particle-particle interactions include the following major components[11-13]:

1. Grain-grain interaction (when two grains are in contact to each other), including the normal force along the line connecting two grain centers and the shear force perpendicular to that line.

2. Cemented bond forces, including the normal and shear forces and the twist and bending moments developed in the "cemented" bonds.

The numerical algorithm used to evolve the model system consists of following steps:

1. For given particle positions in the system, inter-particle interactions are computed, and the resultant forces and moments on all particles are calculated.

2. The particle translational and rotational accelerations are computed from corresponding forces and moments. 
3. A numerical integration is implemented to obtain the new position and velocity at the next time step for each particle based on the current accelerations.

4. Repeat the steps 1-3 with the updated positions and velocities for the next time step.

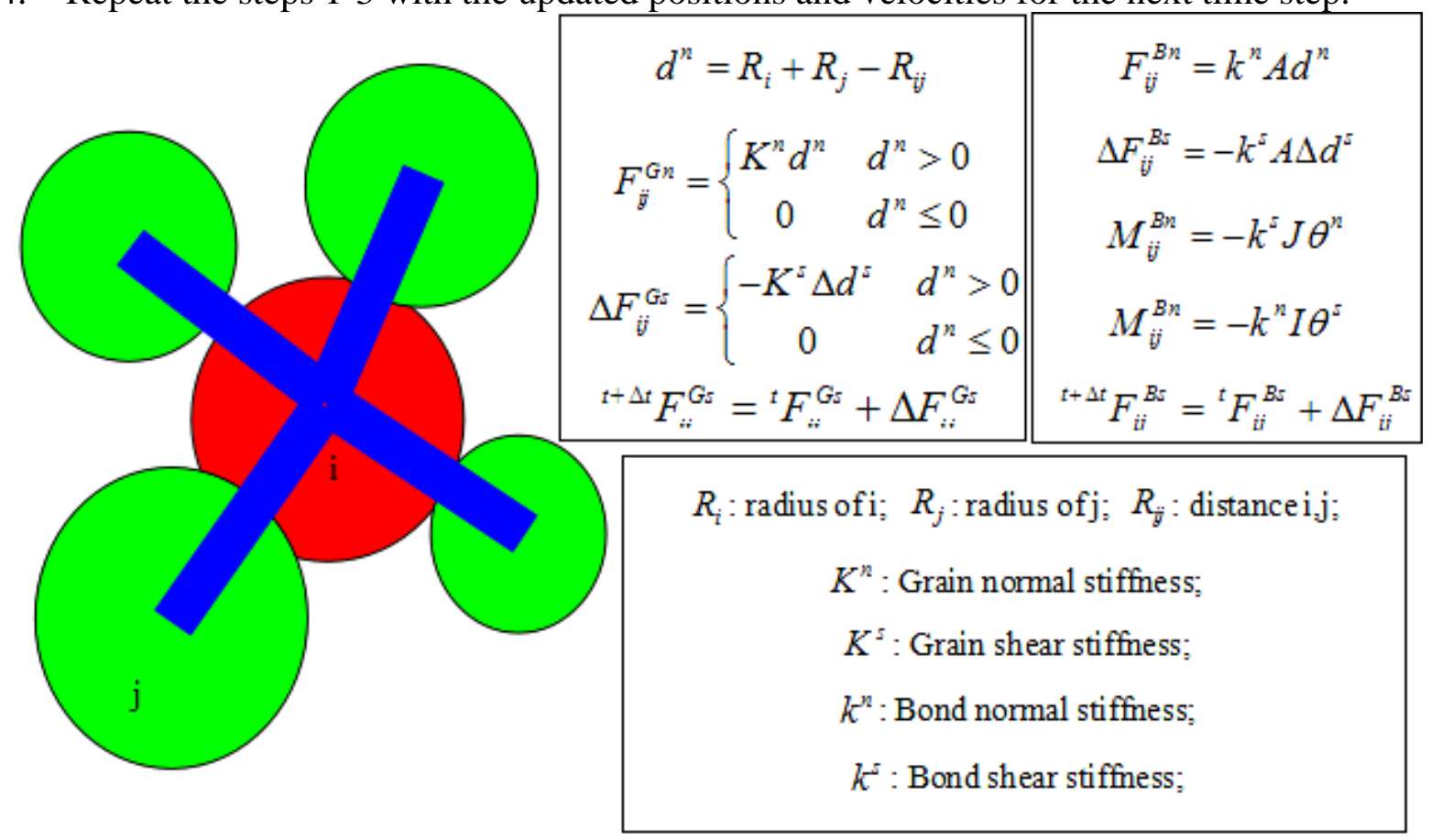

Fig. 1.A schematic plot of DEM particle-particle interaction.

\section{Triaxial Test and Numerical Simulation of Argillaceous Sandstone}

3.1 Preparation and Triaxial tests. The test sample is taken from a dense sandstone layer of a nuclear power project in Guangxi, and the porosity of the rock sample ranges from 5\% to $16 \%$. The instrument adopts the digital controlled electro-hydraulic servo testing machine of Wuhan Institute of Rock and Soil Mechanics, Chinese Academy of Sciences. The collected rock mass is processed into a standard sample of $50 \mathrm{~mm} \times 100 \mathrm{~mm}$. The diameter of the specimen does not exceed $0.30 \mathrm{~mm}$, the unevenness of the two ends is not more than $0.05 \mathrm{~mm}$, the end face is perpendicular to the specimen axis, the maximum deviation does not exceed $0.25^{\circ}$. The molded rock samples are shown in Figure 2

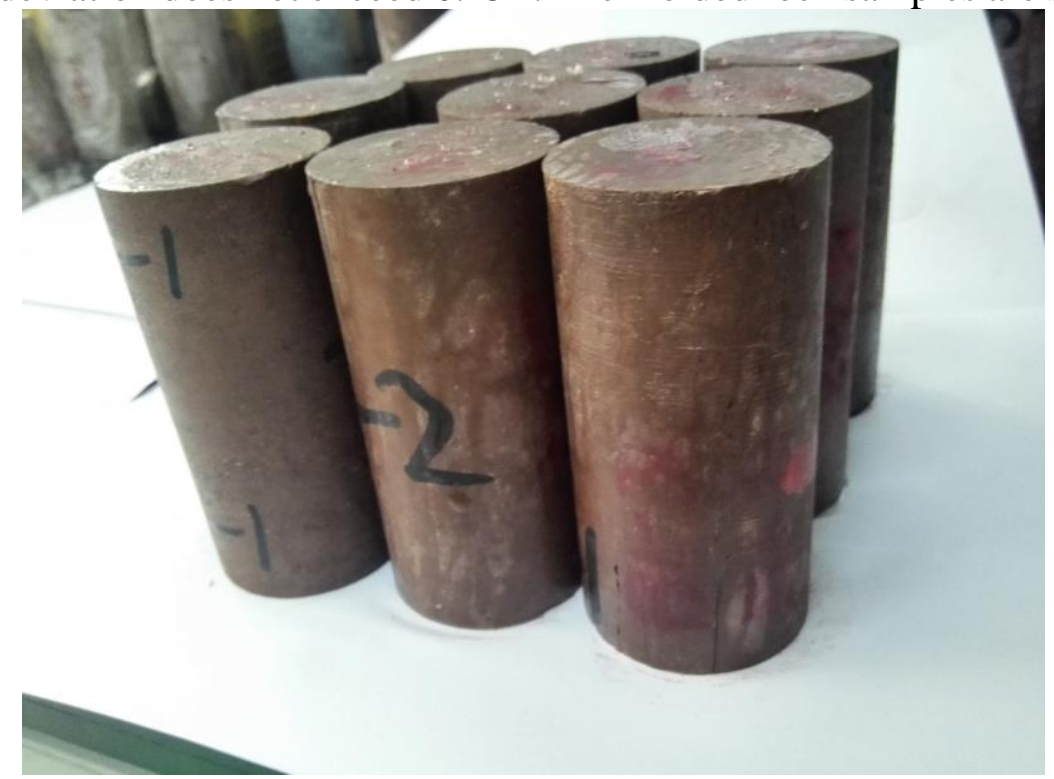

Fig. 2 preparation of argillaceous sandstone samples

3.2 Simulation Model. The numerical simulation of particle flow is different from FLAC, DEC and other numerical simulation software. On the one hand, the discrete element method is a unit-based simulation, and the nature of the particle flow is different from the other two software. On the other 
hand, the discrete element method simulation from the microscopic view, calculation more careful, strict in microscopic parameters of debugging, therefore, microscopic parameters of the discrete element simulation debugging is complex and important part. In this paper, the indoor test parameters substitute into the simulation, the compressive strength of the sample as a measure based on the parameters in order to debug, until a reasonable discrete element response parameters. Therefore, the indoor test parameters correspond to the response parameters one by one but not exactly the same, the sensitivity of the parameters associated with the established numerical model. According to the test parameters to determine the response parameters in the model, after adjustment model, the microscopic parameters of debugging. According to the test parameters to determine the response parameters in the model, after adjustment model, the microscopic parameters of debugging The parallel parameters of the parallel bond model and the new version are roughly the same, but the effect is different, including the maximum and minimum radius of the ball particles, the damping between the particles, the modulus of deformation, the tensile strength of the connection, Of the friction angle, the ratio between the normal stiffness and the tangential stiffness. Microscopic response parameters in Table 1

Table 1 List of the key DEM particle parameters

\begin{tabular}{|c|c|c|c|c|c|c|c|}
\hline $\begin{array}{c}\text { Minimum } \\
\text { particle } \\
\text { size } \\
(\mathrm{mm})\end{array}$ & $\begin{array}{c}\text { Maximum } \\
\text { particle } \\
\text { size } \\
(\mathrm{mm})\end{array}$ & $\begin{array}{c}\text { Normal } \\
\text { strengt } \\
\mathrm{h} \\
(\mathrm{Mpa})\end{array}$ & $\begin{array}{c}\text { Shear } \\
\text { strength } \\
(\mathrm{Mpa})\end{array}$ & $\begin{array}{c}\text { Contact } \\
\text { stiffness } \\
\text { ratio }\end{array}$ & $\begin{array}{c}\text { Poisson } \\
\text { s ratio }\end{array}$ & $\begin{array}{c}\text { Densit } \\
\mathrm{y} \\
(\mathrm{Kg} / \\
\left.\mathrm{m}^{\mathrm{a}}\right)\end{array}$ & $\begin{array}{c}\text { plastic strain } \\
\text { threshold }\end{array}$ \\
\hline 1.2 & 1.8 & 24.2 & 4.5 & 1.25 & 0.22 & 2600 & 0.5 \\
\hline
\end{tabular}

Particle flow code in the microscopic parameters of debugging is the coordination of the overall parameters of debugging, need to find a relatively sensitive and important parameters. In this paper, the tensile strength and cohesion of the two parameters have great influence on the compressive strength obtained by the simulation test, and the modulus parameters and the friction angle have some influence on the compressive strength. Particle flow code operation for the computer requirements is higher, the simulation test, combined with the actual ownership of the computer configuration and parameters, size effects and other factors to consider the number of models of the model set to 11870 , the grain gradation uniform Distribution, the model is shown in Figure 3
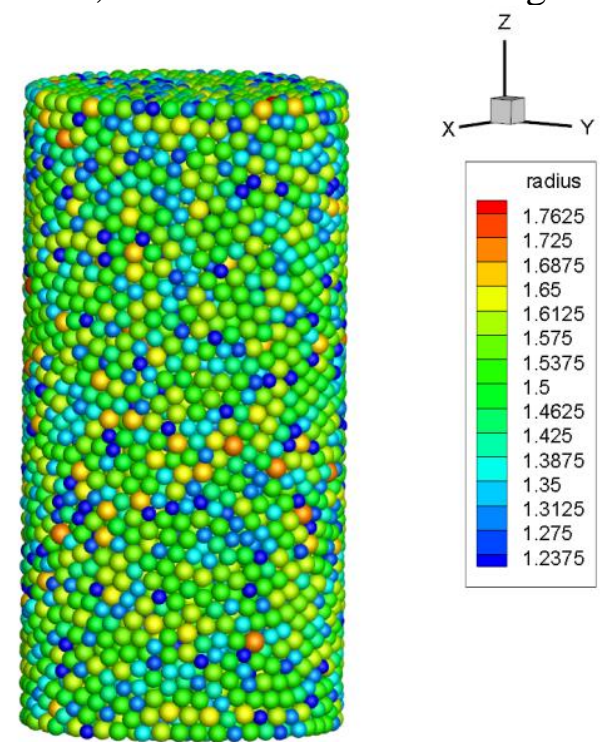

Fig. 3 Numerical model of particle flow code simulation

\section{Result and Discussion}

4.1The comparision of numerical results with experiment results. As shown in Fig 4, the numerical simulation results are consistent with the experimental results, but the bias stress is slightly deviated, and as the confining pressure increases, this deviation is increasing. This is consistent with 
the analysis of Potyondy and Cundall [8]. The stress-strain curves of the three-axis numerical test under the traditional particle flow code model are only consistent with the experimental values under low confining pressure. The main reason is that the basic unit of the particle flow program is a rigid sphere, the shape and arrangement of particles is single, the mutual embedding and bite force between the particles are weak, although the contact bonding is introduced to improve the contact force between the particles. With the increase of axial load, the bond is broken,its contribution to the shear strength is extremely limited, and the actual strength characteristics of sandstone quite different

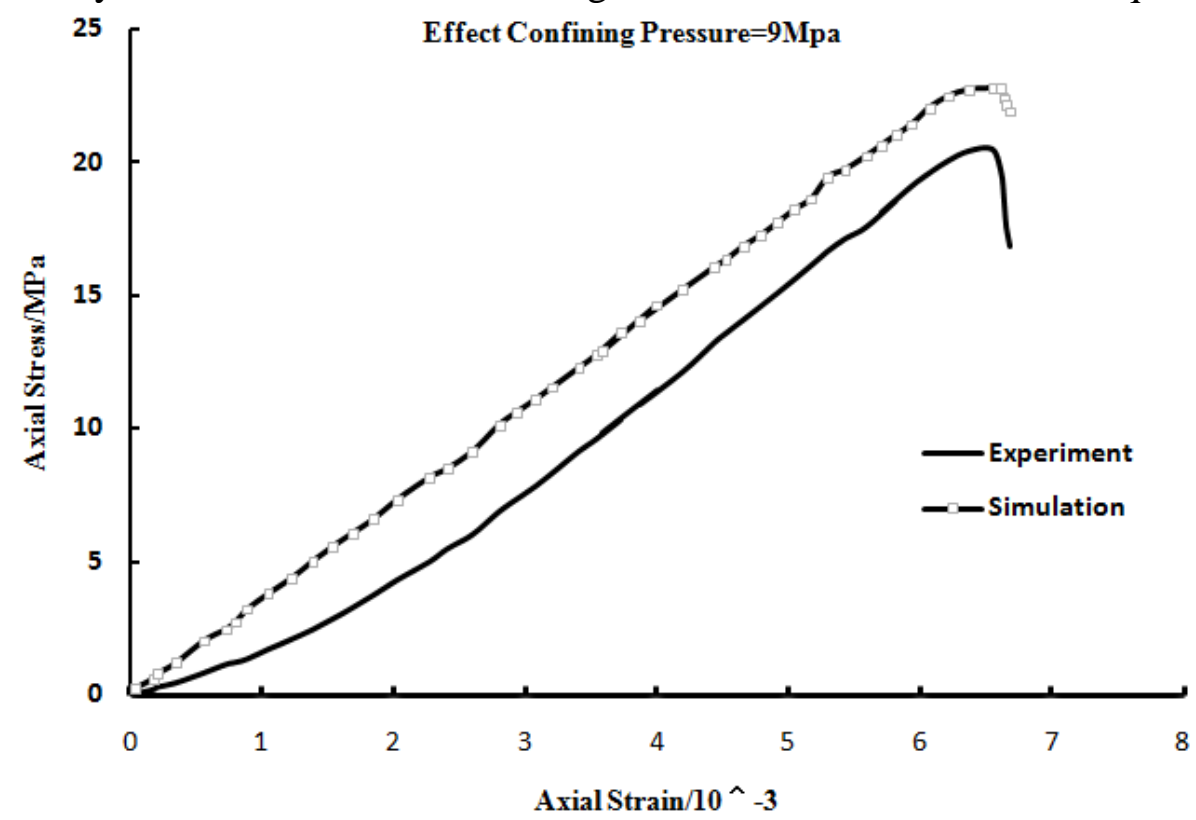

Fig.4 Numerical simulation and experimental stress-strain curves under confining pressure of 9Mpa 4.2 Effect of Friction Coefficient on Strength. As shown in Fig. 5, when the other mesoscopic parameters are constant, the peak strength increases with the increase of the friction coefficient between the particles, and the initial elastic modulus of the material increases slightly, but the residual strength is not very different. When the particles due to rotation or shear force caused by excessive bond damage, the role of the residual contact force at the particle mainly depends on the normal pressure and the friction coefficient between the particles. The frictional force at the contact can suppress the rotation or slip between the particles. The larger the friction coefficient, the greater the interaction between the particles. This process can increase the shear strength of the coarse soil.

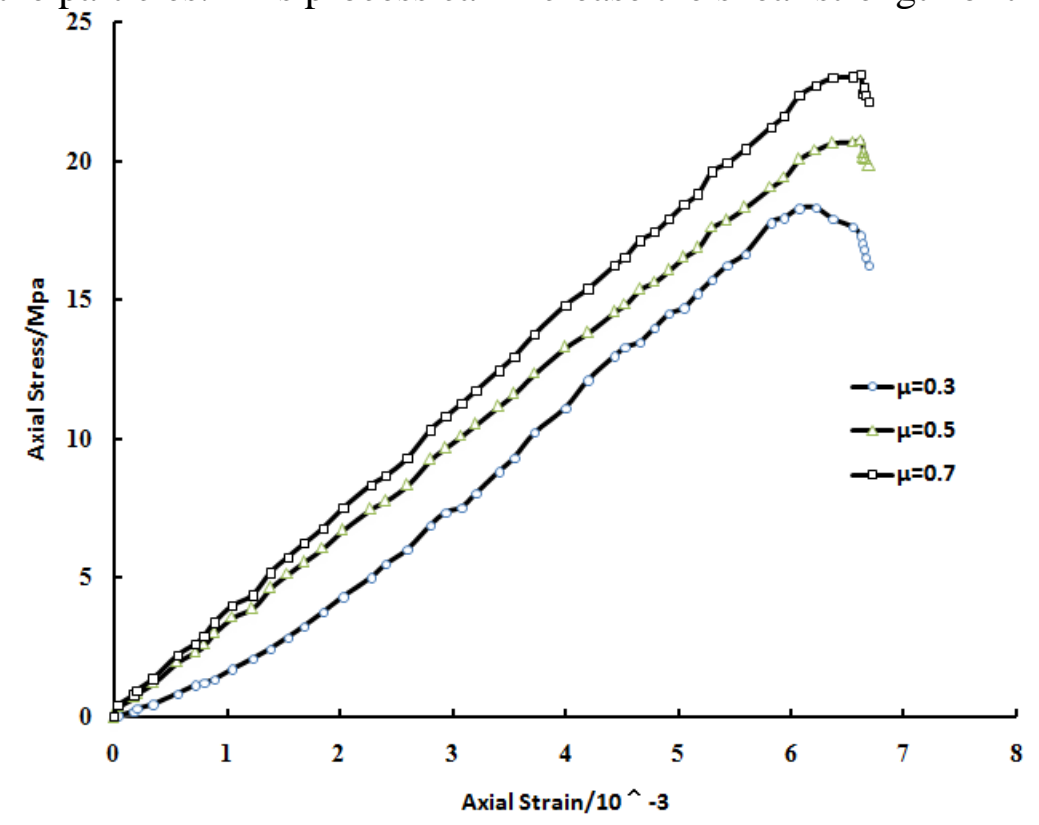

Fig. 5 when the confining pressure 9 Mpa stress-strain curve under different friction coefficient 
4.3 The Influence of Cohesive Strength. The shear surface of the sandstone is not a plane, and its shear strength is not only the internal friction. The coarse particles on the shear plane block shear irregularly curved surfaces, which are arranged in a staggered arrangement to produce shear resistance to increase the strength of the sandstone significantly. The bite force of the sandstone is mainly from the interlocking interdiffusion between the irregular particles, and the basic unit in the model is the ball, which is very different from the actual particle characteristics. In order to simulate the inter-particle bite force, the contact bond model is introduced, between tension and compression effect, the tangential adhesive force not only increases the friction between the particles, but also to contribute to bite force. During the loading process, with the increase of the axial pressure, the contact force between the particles increases, and when the contact force does not reach the bond strength, the intergranular bond force is mainly resisted by the load shear. It can be seen from Fig. 5 that the peak strength increases with the increase of bond strength. When the axial load applied to make the contact force between the particles than the bond strength or due to the rotation of the particles lead to bond damage, the shear strength is only the friction load, so the bond strength of the residual stress is basically the same.

4.4 The Influence of Porosity to Strength. When the specimen is in the initial stress state, the particles are loose and the porosity between the particles is large. As the external force increases during the test, the particles will be filled with each other, so it will shrink. Particles is increased with the increase of axial deformation of the coordination number increase, enhanced constraints among the particles. at this point, the friction between the particles and bite force increases, the corresponding shear strength of increase, reflect on the macro is deviatoric stress increased. With the further increase of stress, the more compact the particles will be between the more tightly, the porosity between the particles is getting smaller and smaller, the coordination number of the particles increases, the friction and bite force of the particles are further increased. At this time to relative movement occurs is not easy. So when the load and then increased to the specimen tends to shear damage, it will force the particles to create movement and rolling conditions, then the particles will be over the adjacent particles, the volume expansion deformation.

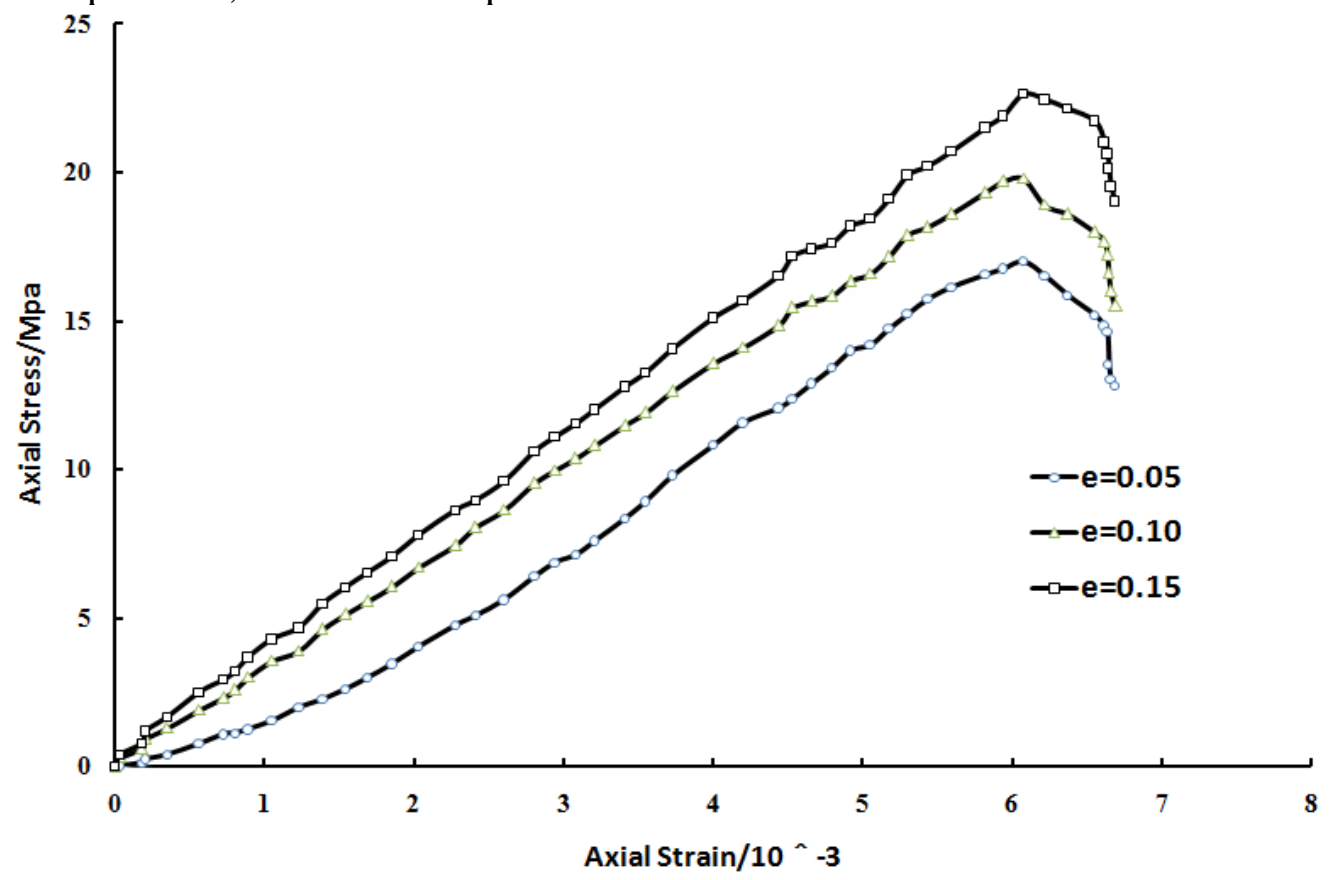

Fig. 6 when the confining pressure 6 Mpa stress-strain curve under different porosity.

\section{Conclusion}

In this paper, three-dimensional model is used to simulate the triaxial test. The influence of some parameters on the shear strength and deformation characteristics of the model soil is studied by the control variable method. We draw the following conclusions . The numerical simulation of the 
three-dimensional model simulates the triaxial test of the argillaceous sandstone, which can flexibly change the test conditions, obtain the test data under different working conditions, overcome the limitation of the capacity of the indoor triaxial test equipment and the discretization of the test results. The stress-strain curves obtained by introducing the contact-bonded constitutive sand flow are consistent with the experimental results, but the partial stress values under different confining pressures are lower than the experimental results. With the increase of the friction coefficient and bond strength in the parallel bond model, the partial stress value of the particle flow model can be improved, but the change is very small. Parameter selecting means particles have a great influence on the macroscopic parameters and a certain law. Reasonable use of these laws, can be adjusted by parameters, and the actual Argillaceous sandstone samples have similar stress-strain relationship, strength and internal friction angle and other macro parameters of the sample.

\section{Acknowledgment}

Thanks to Professor Shouchun Deng, State Key Laboratory of Geomechanics and Geotechnical Engineering, Institute of Rock and Soil Mechanics, Chinese Academy of Sciences, for the careful proofreading. This paper was supported by the Project "Fundamental Theory And Key Technologies of Exploration And Production of Shale Gas (XDB10030400) and the Hundred Talent Program of Chinese Academy of Sciences (Grant No. Y323081C01). We would like to express our greatest gratitude for their generous support. The authors also thank the anonymous referees for their careful reading of this paper and valuable suggestions.

\section{References}

[1] Hong-mei tang, Zhang Jinhao, hong-kai Chen. The deformation and damage of the sandstone under different stress state study [J].The people of the Yangtze river, 2014, (12) : $81 \sim 84$.

[2] Tielin Han, Yunsheng Chen, Yongjun Song, etc. The mechanical properties of sandstone under different stress path test research Investigate [J]. Journal of rock mechanics and engineering, 2012, 31 (2): $3659 \sim 3966$.

[3] Xi-liang liu, Hou Yuan, xu, et al. A deep experimental study on the mechanics characteristics of fine sandstone [J].Journal of henan university of technology (natural science edition), 2015 (4) : $556 \sim 561$.

[4] Song Han, mei-feng CAI. The mechanical properties of in-situ stress field of deep sandstone change [J]. Journal of coal,2007, 32 (6) : $570 \sim 572$.

[5] Wu Gang, patriotic xing, zhang lei. The mechanical properties of sandstone after high temperature $[\mathrm{J}]$. Journal of rock mechanics and engineering, 2007, 26 (10) : $2110 \sim 2116$.

[6] Anbin Zhang Yanbo Zhang, Xiangxin Liu etc. The water effects on the physical and mechanical properties of argillaceous siltstone experimental study [J]. Journal of coal science and technology, 2015 (8) : $67 \sim 71$.

[7] lu wang, jian-feng liu,JianLiang Pei etc., fine permeability sandstone damage process and acoustic emission characteristics experimental study $[\mathrm{J}]$. Journal of rock mechanics and engineering, 2015 (1-6) : $2909 \sim 2914$.

[8] Kang Han. Different size sandstone triaxial compression mechanical properties experimental study [J]. Journal of subgrade engineering, 2013 (6) : $94 \sim 96$.

[9] Zou Hang, jian-feng liu, yu, etc. Different granularity sandstone mechanics and seepage characteristics of experimental study [J]. Journal of geotechnical engineering, 2015, 5 (8) : 1462 $\sim 1468$. 
[10] xian-shan liu, xu Ming. Particle size distribution on the mechanical properties of cementation sandstone influence [J]. Journal of China petroleum university (natural science edition), 2014, 42 (5) : $142 \sim 148$.

[11] D.O. Potyondy, P.A. Cundall, A bonded-particle model for rock, International Journal of Rock Mechanics and Mining Sciences, 41 (2004) 1329-1364.

[12] P.A. Cundall, Formulation of a three-dimensional distinct element model-Part I. A scheme to detect and represent contacts in a system composed of many polyhedral blocks, International

[13] P.A. Cundall, O.D.L. Strack, Modeling of Microscopic Mechanisms in Granular Material, in: T.J. James, S. Masao (Eds.) Studies in Applied Mechanics, Elsevier, 1983, pp. 137-149.Journal of Rock Mechanics and Mining Sciences \& Geomechanics Abstracts, 25 (1988) 107-116. 